\title{
Topological Quantum Field Theories, Strings and Orbifolds.
}

\author{
Ernesto Lupercio and Bernardo Uribe
}

Dedicated in memoriam to Prof. Raoul Bott.

\begin{abstract}
In this article, written primarily for physicists and geometers, we introduce the notion of TQFT, orbifold, and then we survey the construction of TQFTs originating from orbifolds such as Chen-Ruan cohomology and orbifold string topology.
\end{abstract}

\section{Contents}

1. Topological Quantum Field Theories 1

2. Group Actions 9

3. Orbifolds. 11

4. Groupoids 16

5. Moduli Spaces 18

6. Almost Free Lie Group Actions. 19

7. The Algebraic Topology of Orbifolds. 21

8. Loop Orbifolds 22

9. Orbifold TQFTs 24

10. Acknowledgments 26

References 26

\section{Topological Quantum Field Theories}

In quantum mechanics one encounters the following situation.

EXAMPLE 1.0.1. Let $M$ be a Riemannian manifold (you may want to think that it is $M=\mathbb{R}^{3}$ ).

Given two points in $M$, say $p$ and $q$, we would like to compute the probability that a particle that starts in $p$ lands in $q$ after certain amount of time $T$. The answer is, of course, zero, but we can nevertheless still ask what is the probability that the particle will be at a distance less than $\epsilon$ from $q$.

The first author was partially supported by CONACYT-México.

(C)0000 (copyright holder) 
Feynman gave a remarkable formula for the probability 11. Say that $\phi$ is the initial probability distribution for the position of the particle at $t=0$ (meaning that $\int_{U}\left|\phi_{0}\right|$ is the probability that the particle is in $U$ at $t=0$ ). Then the probability distribution $\phi_{T}$ for the position at $t=T$ is given by the path integral

$$
\phi_{T}(q)=\int_{\mathcal{P}_{q}} \phi_{0}(\gamma(0)) e^{-i \hbar S(\gamma)} \mathcal{D} \gamma
$$

where

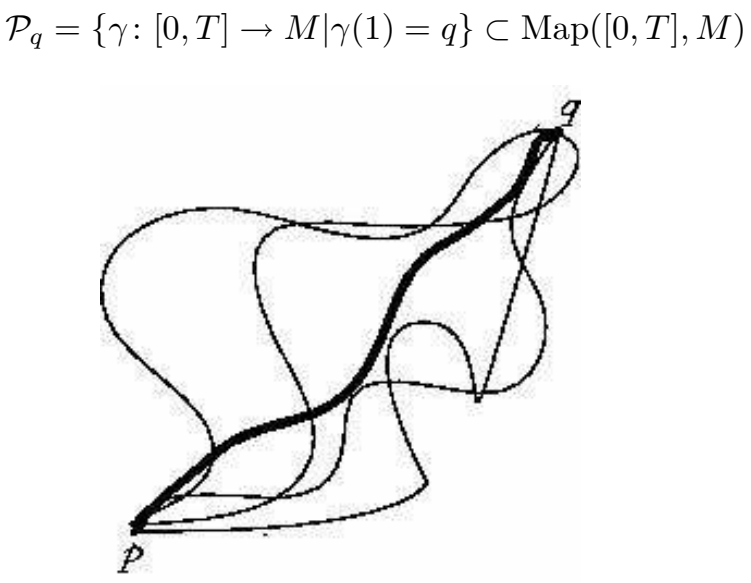

and

$$
S(\gamma)=\frac{1}{2} \int_{0}^{T}\left|\gamma^{\prime}(t)\right|^{2} d t
$$

In the picture we stress the classical (Euler-Lagrange) path minimizing $S$.

Moreover, if we think of $\left|\phi_{t}\right\rangle=\phi(q, t)$ as a one parameter family of vectors (kets) in $\mathcal{H}=\operatorname{Map}(M, \mathbb{C})$ (usually thought of as a Hilbert space) then we have that the main result of Feynman in this case is that $\phi$ satisfies the Schrödinger equation.

We can try to extract the formal structure behind formula 1.0.1 as follows.

Consider $P_{T}$ to be a compact 1-dimensional manifold with boundary (namely $P=[0, T])$. We define the fields on a 1-manifold $Y$ to be

$$
\mathcal{F}(Y)=\operatorname{Map}(Y, M),
$$

the moduli space ${ }^{1}$ of all maps from $Y$ to $M$. We will divide the boundary of $Y$ into two portions that we will call the incoming and outgoing boundaries

$$
\partial Y=\partial_{0} Y \coprod \partial_{1} Y
$$

As part of the structure we need an action map

$$
S_{Y}: \mathcal{F}(Y) \rightarrow \mathbb{R}
$$

which in our case could be given by

$$
S(\gamma)=\frac{1}{2} \int_{Y}\left|\gamma^{\prime}\right|^{2}
$$

We have the following properties:

\footnotetext{
${ }^{1}$ We will return to the subject of moduli spaces below. They are often orbifolds.
} 
i) We have restriction maps (forming a correspondence)

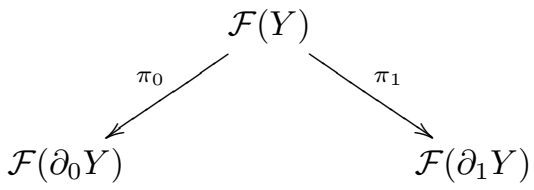

ii) Whenever we have $Y=Y^{\prime} \cup Y^{\prime \prime}$ where $Y^{\prime} \cap Y^{\prime \prime}=\partial_{1} Y^{\prime}=\partial_{0} Y^{\prime \prime}$

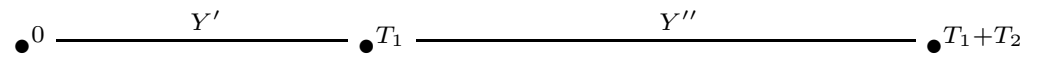

then

$$
S_{Y}(\gamma)=S_{Y}^{\prime}\left(\left.\gamma\right|_{Y^{\prime}}\right)+S_{Y}^{\prime \prime}\left(\left.\gamma\right|_{Y^{\prime \prime}}\right)
$$

iii) We have the following pull-back diagram ${ }^{2}$

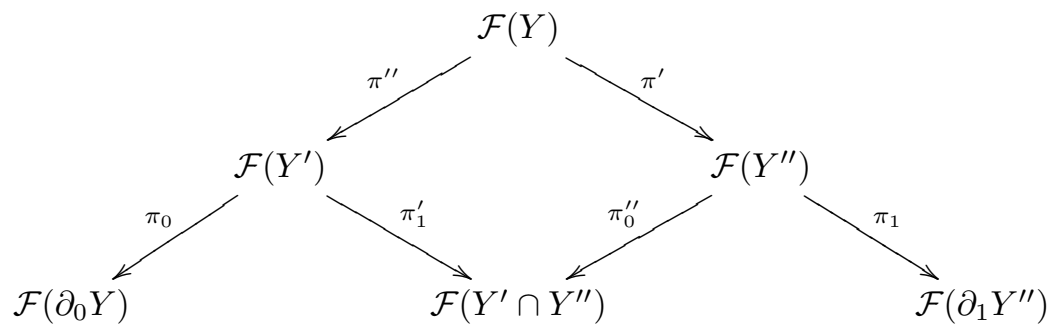

iv) The initial ket $\left|\phi_{0}\right\rangle$ evolves along $Y$ according to the formula

$$
\left.\left|\phi_{T}\right\rangle=\left(\pi_{T}\right) !\left(\pi_{0}^{*}\left(\left|\phi_{0}\right\rangle\right) \cdot e^{-i \hbar S}\right)\right)
$$

We will call this the pull-push evolution formula. It is the fundamental formula for what follows and requires some clarification.

- $\left|\phi_{0}\right\rangle \in \mathcal{H}$ can be seen as an element in $\operatorname{Map}\left(\mathcal{F}\left(\partial_{0} Y\right), \mathbb{C}\right)$ for $\partial_{0} Y=\bullet$ a point and hence $\mathcal{F}\left(\partial_{0} Y\right)=\mathcal{F}(\bullet)=\operatorname{Map}(\bullet, M) \cong M$.

- $\pi_{0}^{*}\left(\left|\phi_{0}\right\rangle\right)$ is an element in $\operatorname{Map}(\mathcal{F}(Y), \mathbb{C})$. In fact when we evaluate at $\gamma \in \mathcal{F}(Y)$ we get $\left(\pi_{0}^{*}\left(\left|\phi_{0}\right\rangle\right)\right)(\gamma)=\phi_{0}\left(\gamma\left(\partial_{0} Y\right)\right)=\phi_{0}(\gamma(0))$.

- $\left(\pi_{1}\right) !: \operatorname{Map}(\mathcal{F}(Y), \mathbb{C}) \rightarrow \operatorname{Map}(\mathcal{F}(\bullet), \mathbb{C})$ is the map that integrates over the fiber of $\pi_{1}: \mathcal{F}(Y) \rightarrow \mathcal{F}(\bullet)$ (which in this example is the path space $\mathcal{P}_{q}$ and therefore it is given by a path integral). Namely

$$
\left(\left(\pi_{1}\right) !(\Phi)\right)(q)=\int_{\mathcal{P}_{q}} \Phi(\gamma) \mathcal{D} \gamma
$$

- You may want to think of the exponential term as a sort of Chern class for a line bundle over $\mathcal{F}(Y)$. It causes the integral to become oscillatory, and when $\hbar$ approaches 0 , stationary phase approximation makes the probability that the particle travels the classical (EulerLagrange) path approach to 1 . Feynman designed it with this express purpose [1].

- Formula 1.0.2 is in fact exactly equivalent to formula 1.0.1

\footnotetext{
${ }^{2}$ The fact that this diagram is cartesian implies that we do have a 1-parameter action on $\mathcal{H}=\operatorname{Map}(M, \mathbb{C})=\operatorname{Map}(\mathcal{F}(\bullet), \mathbb{C})$.
} 
The algebraic abstract structure that we will extract from this is the following. Define

$$
\mathcal{H}_{Y}:=\operatorname{Map}(\mathcal{F}(Y), \mathbb{C})
$$

then we have

a) We will write $\mathcal{H}$ for $\mathcal{H}(\bullet)$. To every 0 -dimensional manifold we have associated a vector space $\mathcal{H}$.

b) To every 1-dimensional manifold (say of length $T$ ) we have associated a linear operator

$$
\begin{aligned}
\Psi_{T}: \mathcal{H} & \rightarrow \mathcal{H} \\
\Psi_{T}\left(\phi_{0}\right) & =\phi_{T} .
\end{aligned}
$$

c) Whenever we glue 1-manifolds, we compose the corresponding linear operators. Namely we have homomorphism from $\mathbb{R}$ to GL $(\mathcal{H})$.

The field theory just described is not topological, for the operators depend on the length $T$ of the 1-manifold. In a topological theory the operators are independent on the geometry of the 1-manifold and only depend on their topology (hence we only have two operators, the one associated to the interval, and the number associated to the circle).

Here we should mention that in string theory we usually start by assuming that rather than point particles interacting at singular points, we consider extended strings as in the following picture.
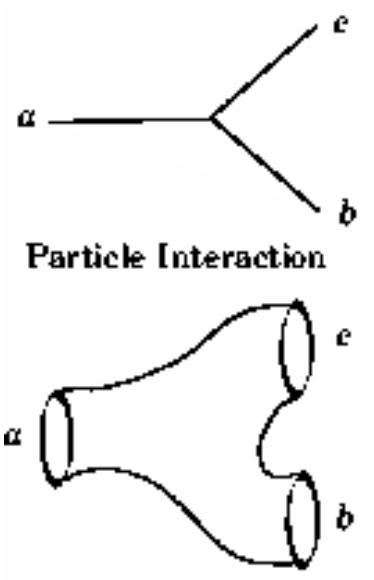

String Interaction

In the picture we have a particle $a$ scattering in to a pair of particles $b$ and $c$, and the corresponding situation with a string scattering. You should think of this picture as living inside the ambient space time $M$. Notice that the string interaction has no singularity.

Traditionally one thinks of $M$ as a smooth manifold, for example in general relativity. Later in this paper we will think instead that the ambient space-time is an orbifold $\mathrm{X}$. While a (parameterized) string on a manifold can be modeled by an element of the free loop space

$$
\gamma \in \mathcal{L} M=\operatorname{Map}\left(S^{1}, M\right)
$$


namely a piecewise smooth map form the circle to $M$, in an orbifold the definition of a loop is more intricate, we will come on this issue later.

Let us remember that a $(n+1)$-dimensional TQFT is a functor $H$ from the category of smooth manifolds and diffeomorphisms to the category of vector spaces that additionally assigns to each cobordism $Y$ between two smooth manifolds $M$ and $N$ (namely $\partial Y=M \amalg-N$ ), a linear mapping $\Psi_{Y}: H(M) \rightarrow H(N)$. Both $H$ and $\Psi$ should be compatible with all the obvious structures including gluing of cobordisms $\left[\right.$ 3, 10]. Traditionally whenever $Y$ is boundaryless the map $\Psi_{Y}: \mathbb{C} \rightarrow \mathbb{C}$ is identified with a number $Z(Y)$. In particular we have that the following properties hold,

- $\Psi_{\varnothing}=\mathbb{C}$.

- $\Psi_{(M \times I)}=\mathbf{1}_{H(M)}$, if $I=[0,1]$.

- $H(M \amalg N) \cong H(M) \otimes H(N)$.

- $H(-M) \cong H(M)^{*}$.

- $\operatorname{dim} H(M)=Z\left(M \times S^{1}\right)$.

- Whenever we have $Y=Y^{\prime} \cup Y^{\prime \prime}$ where $Y^{\prime} \cap Y^{\prime \prime}=\partial_{1} Y^{\prime}=\partial_{0} Y^{\prime \prime}$ then

$$
\Psi_{Y}=\Psi_{Y^{\prime \prime}} \circ \Psi_{Y^{\prime}}
$$

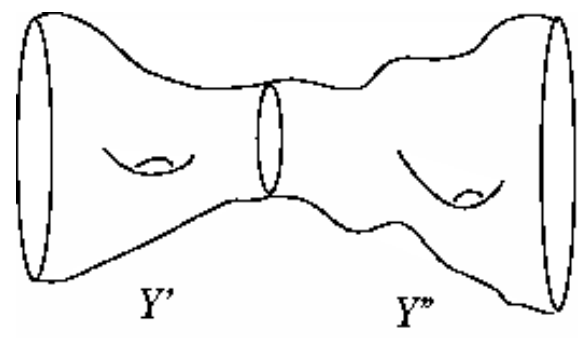

We will also need the definition of a Frobenius algebra. A finite-dimensional commutative algebra $A$ over $\mathbb{C}$ with a unit, together with a linear map $\theta: A \rightarrow \mathbb{C}$ such that $(x \mid y) \mapsto \theta(x y)$ is a nondegenerate bilinear form on $A$ is called a Frobenius algebra. In this case $\theta$ is called a nonsingular trace [1, 27.

It is well-known that to have $(1+1)$-dimensional field theory is the same as having a Frobenius algebra. The basic idea is to set $A=H\left(S^{1}\right)$, the product corresponding to the pair of pants $Y_{P}$, and the trace to a disk $Y_{D}$ thought of as having one boundary component coming in and none coming out.

ExAmple 1.0.2. There is another example afforded to us by Poincaré duality. This model written $\left(H^{M}, \Psi^{M}, Z^{M}\right)_{1+1} \cong\left(A_{M}, \theta_{M}\right)$ depends only of a fixed oriented compact closed smooth manifold $M$ and lives in dimension $1+1$. Let $\operatorname{Map}^{\odot}(Y, M)$ be the space of constant maps from $Y$ to $M$. Clearly if $Y$ is connected (and nonempty), $\operatorname{Map}^{\odot}(Y, M) \cong M$ and in fact this last homeomorphism is given by the map

$$
\mathrm{ev}_{y}: \operatorname{Map}^{\odot}(Y, M) \rightarrow M
$$

that evaluates at $y \in Y$. For $Z \subset Y$ we will write $\operatorname{ev}_{Z}: \operatorname{Map}^{\odot}(Y, M) \rightarrow \operatorname{Map}^{\odot}(Z, M)$ to be the restriction map defined by $\operatorname{ev}_{Z}(f)=\left.f\right|_{Z}$.

In this theory the fields are

$$
\mathcal{F}(Y)=\operatorname{Map}^{\odot}(Y, M)
$$


namely the moduli space of constant maps from $Y$ to $M$. We consider $Y$ to be $(1+1)$-dimensional. Notice that

$$
\operatorname{Map}^{\odot}(Y, M)=M \times M \times \cdots \times M
$$

where the product contains as many copies of $M$ as connected components has $Y$. Consider now the situation in which $Y=P$ a 2-dimensional pair-of-pants (a 2 -sphere with three small discs removed) with two incoming boundary components and one outgoing, and $M$ is an oriented compact closed smooth manifold. Let $a, b$ and $c$ be three boundary components $P$ each one diffeomorphic to $S^{1}$.

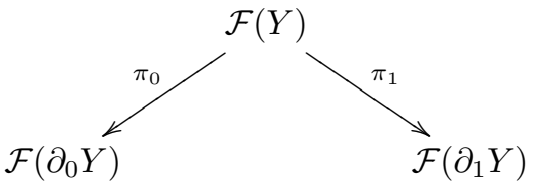

that is to say

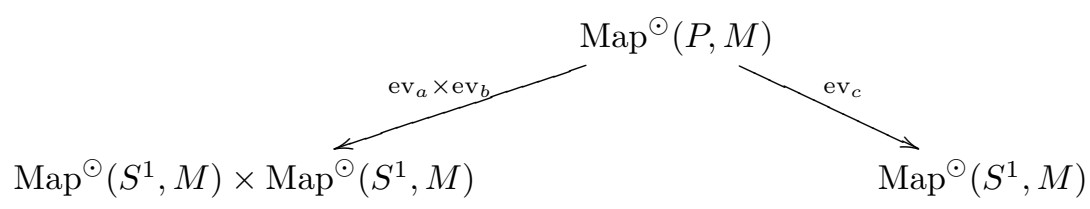

which becomes thus

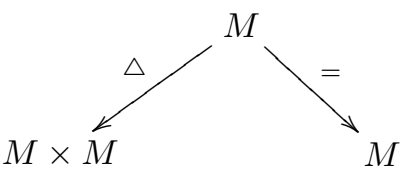

and indeed, since that is a smooth correspondence of degree $-d$ we have that

$$
\triangle_{!}=e v_{c} \circ\left(\mathrm{ev}_{a} \times \mathrm{ev}_{b}\right)_{!}: H_{*}(M) \otimes H_{*}(M) \rightarrow H_{*-d}(M)
$$

is the induced homomorphism of degree $-d$ in homology. Namely, the Feynman pull-push evolution for a pair of pants in this field theory is simply the intersection product in homology.

We could have used the space 8 consisting of the wedge of two copies of $S^{1}$ instead of $P$ (they are after all homotopy equivalent, we can define $\mathrm{ev}_{c}$ by choosing a quotient map $c \rightarrow \mathbf{8}$ identifying two points of $c$ ). Notice that by using pairs-ofpants we can recover any compact oriented 2-dimensional cobordism $Y$ which is not boundaryless. In fact by using correspondences we can recover $\Psi_{Y}^{M}$ for all $Y$ that has at least one outgoing boundary component. In a sense correspondences encode a big portion of Poincaré duality this way, the so-called positive boundary sector of the TQFT.

For this model we have,

- $A_{M}=\mathcal{H}(\bullet)=H_{*}(M)$ (the homology of $M$ which is graded).

- The mapping associated to the pair of pants

$$
A_{M} \otimes A_{M} \rightarrow A_{M}
$$

is the intersection product on the homology of the manifold (and is of degree $-d$ ).

- The trace is defined as $\theta_{M}: A_{M}=H_{*}(M) \rightarrow H_{*}(\bullet) \cong \mathbb{C}$. The nondegeneracy of the trace is a consequence of Poincaré duality. 
It may be instructive to see how the Pontrjagin-Thom construction and the Thom isomorphism can be used to induce the map 1.0.4. That basic idea is to use the diagonal map

$$
\begin{gathered}
\triangle: M \rightarrow M \times M . \\
m \mapsto(m, m)
\end{gathered}
$$

The product on $A_{M}$ is precisely the Gysin map $\triangle$ ! which can be defined using integration over the fiber, or as follows. It is not hard to verify that the normal bundle $\nu$ of $M=\triangle(M)$ in $M \times M$ is isomorphic to the tangent bundle $T M$ of $M$. Let us write $M_{\epsilon}$ a small neighborhood of $M$ in $M \times M$, and $M^{T M}$ the Thom space on $T M$. Then we have a natural map

$$
M \times M \longrightarrow M \times M /\left(M \times M-M_{\epsilon}\right)=M^{T M}
$$

which by the use of the Thom isomorphism induces

$$
\triangle_{!}: H_{*}(M) \otimes H_{*}(M) \longrightarrow H_{*-d}(M)
$$

as desired.

Example 1.0.3. This is a famous example due to Chas and Sullivan [4]. Following Cohen and Jones [7 we do something rather drastic now and let the maps roam free, namely we write the correspondence 1.0 .3 but with the whole mapping spaces rather than just the constant maps.

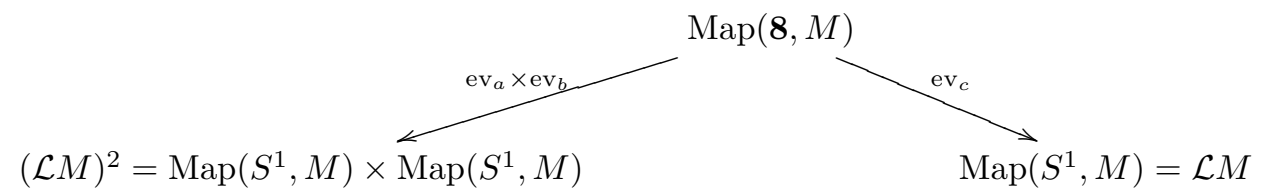

which is a degree $-d$ smooth correspondence. We must replace the pair of pants $P$ for the figure eight space $\mathbf{8}$ in order to ensure that $\operatorname{Map}(\mathbf{8}, M) \rightarrow \mathcal{L} M \times \mathcal{L} M$ is a finite codimension embedding. This in turns implies the existence of the Gysin map

$$
\left(\mathrm{ev}_{a} \times \mathrm{ev}_{b}\right) !: H_{*}(\mathcal{L} M \times \mathcal{L} M) \rightarrow H_{*-d}(\operatorname{Map}(\mathbf{8}, M)) .
$$

The induced map in homology

$$
\text { - : } H_{*}(\mathcal{L} M) \otimes H_{*}(\mathcal{L} M) \rightarrow H_{*-d}(\mathcal{L} M)
$$

is called the Chas-Sullivan product on the homology of the free loop space of $M$. From the functoriality of correspondences it isn't hard to verify that the product is associative.

Chas and Sullivan proved more, by defining a degree one map $\Delta: H_{*}(\mathcal{L} M) \rightarrow$ $H_{*+1}(\mathcal{L} M)$ given by $\Delta(\sigma)=\rho_{*}(\theta \otimes \sigma)$ where $\rho: S^{1} \times \mathcal{L} M \rightarrow \mathcal{L} M$ is the evaluation map and $\theta$ is the generator of $H^{1}\left(S^{1}, \mathbb{Z}\right)$, they proved that $\left(H_{*}(M), \bullet, \Delta\right)$ is a Batalin-Vilkovisky algebra, namely

- $\left(H_{*-d}(M), \bullet\right)$ is a graded commutative algebra.

- $\Delta^{2}=0$

- The bracket $\{\alpha, \beta\}=(-1)^{|\alpha|} \Delta(\alpha \bullet \beta)-(-1)^{|\alpha|} \Delta(\alpha) \bullet \beta-\alpha \bullet \Delta(\beta)$ makes $H_{*-d}(M)$ into a graded Gerstenhaber algebra (namely it is a Lie bracket which is a derivation on each variable). 
This statement amounts essentially to the construction of $\Psi_{Y}^{\mathcal{L}} M$ for all positive boundary genus zero $(1+1)$-dimensional cobordisms $Y$ due to a theorem of Getzler (cf. [12]). The case of positive genus has been studied by Cohen and Godin [6]

ExAMPLE 1.0.4. The Gromov-Witten invariants introduced by Ruan in [22] can be understood in terms of a field theory 21. Now we consider a Riemmann surface $Y=\Sigma_{g}$ of genus $g$ with $k$ marked points. These marked points will take the place of $\partial_{0} Y$ and for simplicity we will not consider outgoing boundary for now.

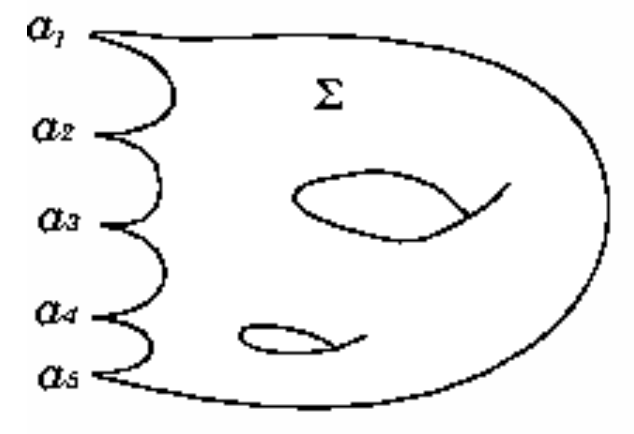

Riemann Surface with five marked points

In this $(1+1)$-dimensional quantum field theory we start by considering a fixed symplectic manifold $(M, \omega)$. The space of fields is given (roughly speaking) by the space of $J$-holomorphic maps on the class $\beta \in H_{2}(M)$,

$$
\mathcal{F}(Y)=\mathcal{M}_{\Sigma}=\operatorname{Hol}_{\beta}(\Sigma, M)=\left\{f \in \operatorname{Hol}(\Sigma, M) \mid f_{*}[\Sigma]=\beta\right\},
$$

If we denote by $\operatorname{ev}_{i}: \mathcal{M}_{\Sigma} \rightarrow M$ the evaluation map at $a_{i} \in \Sigma$, then we have the correspondence diagram

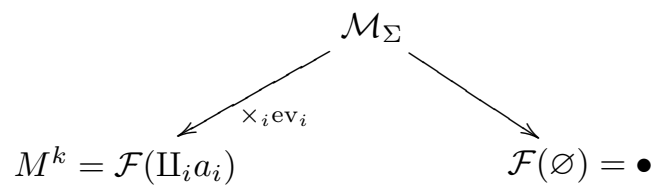

Given $k$ cohomology classes $u_{1}, \ldots, u_{k} \in H^{*}(M)$ we can let them evolve according to Feynman's pull-push formalism to obtain the corresponding Gromov-Witten invariant

$$
\Phi_{g, \beta, k}\left(u_{1}, \ldots, u_{k}\right)=\int_{\mathcal{M}_{\Sigma}} \mathrm{ev}_{1}^{*} u_{1} \wedge \ldots \wedge \mathrm{ev}_{k}^{*} u_{k}
$$

Here we should mention two important technical points regarding the moduli space $\mathcal{M}_{\Sigma}$. Firstly Kontsevich [13] discovered that the most convenient space for defining this field theory is the moduli space of stable maps (where at most ordinary double points are allowed, and with finite automorphism groups). The moduli space turns out to be an orbifold, not a manifold. We will return to the definition of an orbifold later.

Secondly, the moduli space does not quite have a fundamental class (that we require to do the integration). The problem is that roughly speaking $\mathcal{M}$ is given as the intersection of two submanifolds (equations) $N_{1}$ and $N_{2}$ of a larger manifold $V$ (taking only two is possible by using the diagonal map trick, namely $N_{1} \cap \ldots \cap N_{r}=$ 
$\left.\left(N_{1} \times \cdots \times N_{r}\right) \cap \triangle\left(V^{r}\right)\right)$. Often this intersection is not transversal. Therefore rather than a tangent we have a virtual tangent bundle (in $K$-theory)

$$
[T \mathcal{M}]^{\text {virt }}=\left.\left[T N_{1}\right]\right|_{\mathcal{M}}+\left.\left[T N_{2}\right]\right|_{\mathcal{M}}-\left.[T V]\right|_{\mathcal{M}}
$$

whose orientation (in cohomology, K-theory, complex cobordism) is called the virtual fundamental class $[\mathcal{M}]^{\text {virt }}$. The corrected formula for the Gromov-Witten invariants is then

$$
\Phi_{g, \beta, k}\left(u_{1}, \ldots, u_{k}\right)=\int_{\left[\mathcal{M}_{\Sigma}\right]^{\mathrm{virt}}} \operatorname{ev}_{1}^{*} u_{1} \wedge \ldots \wedge \mathrm{ev}_{k}^{*} u_{k} .
$$

EXAMPLE 1.0.5. Floer theory is also a quantum field theory. Now we consider $Y=\Sigma_{g, k}$ to be a genus $g$ Riemann surface with $k$ small discs removed.

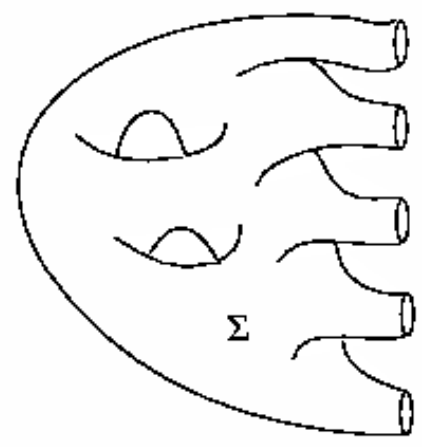

The fields are again holomorphic mappings $\mathcal{F}(Y)=\mathcal{M}_{\Sigma}$.

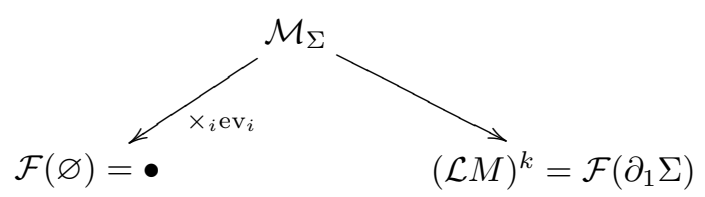

In this case rather than simply considering the homology of $\mathcal{L} M$ we consider its semi-infinite (co)homology. This means that we consider the homology of cycles that are half-dimensional in $\mathcal{L} M$. The semi-infinite $\left(\right.$ co)homology $H_{*}^{\mathrm{si}}(\mathcal{L} M)$ is also known as the Floer (co)homology $H F_{*}(M)$.

Cohen's conjecture states that the quantum field theory of Chas-Sullivan on a manifold $M$ is isomorphic to the Floer quantum field theory of $T^{*} M$ (which is always symplectic).

\section{Group Actions}

Given a space $M$ we often want to study all its self-transformations that preserve some of its properties. Often such transformations are called symmetries and often they are also called automorphisms.

EXAmple 2.0.6. Consider a triangle $T$ as a subset of $\mathbb{R}^{2}$. We may ask how many mappings $g: T \rightarrow T$ there are with the property

$$
d(x, y)=d(g(x), g(y))
$$


for every pair of points in the triangle, where $d$ denotes the usual distance. Such a map is called an isometry of the triangle.

The answer of course depends very much on the triangle.

- If the triangle is scalene only the identity is an isometry of $T$.

- If the triangle is isosceles then there are two such isometries.

- If the triangle is equilateral there are six isometries of $T$.

This can be verified by noticing that an isometry is completely determined by its restriction to the vertices.

Here, as we all know, we can take a remarkable conceptual leap: we decide to remember how the different symmetries interact rather than the symmetries themselves. For this we observe that

- If $g$ and $h$ are symmetries of $T$ so is $g \circ h=g h$.

- $(g h) k=g(h k)$

- There is always the identity symmetry $1_{T}$.

- Given a symmetry $g$ there is another symmetry $k$ such that $g k=k g=1_{T}$.

This motivates the definition of (abstract) group [15. A group is a set of things, together with a composition law that satisfies all the previous axioms. We say for example that the isometries of $T$ form a group.

Once we have this definition we end up with groups that are (at first) not naturally the symmetries of anything. For example, the fundamental group of a space $X$ is at first an abstract group formed with homotopy classes of paths. In this case it may come as a surprise to learn that $\pi_{1}(X)$ in fact acts as some sort of symmetry, namely as deck transformations of the universal cover $M=\widetilde{X}$. It is often important to realize that an (abstract) group is indeed a group of transformations of some space $M$.

Definition 2.0.7. We say that the group $G$ acts on the object $M$ if we are given a homomorphism

$$
\psi: G \rightarrow \operatorname{Aut}(M),
$$

Namely, for every $g \in G$ and every $m \in M$ we have

- $m g=\psi(g)(m) \in M$ such that

- $m 1_{M}=m$

- $(m g) h=m(g h)$

Definition 2.0.8. We say that the group $G$ acts effectively on the object $M$ if $\psi: G \rightarrow \operatorname{Aut}(M)$ is injective, namely for all $g \in G, g \neq 1$ there is an $m \in M$ so that $m g \neq m$.

Definition 2.0.9. The equivalence relation induced by the action of $G$ on $M$ is the relation generated by

$$
x \sim x g .
$$

The quotient $M / \sim$ is also written

$$
M / G \text {. }
$$

The equivalence classes of this relation are called the orbits of the action. They are written

$$
[m]=m \cdot G=\{m g \mid g \in G\} .
$$

If there is only one equivalence class (orbit) for the action we say that $G$ acts transitively on $M$. 
Definition 2.0.10. The stabilizer subgroup of $m \in M$ is

$$
G_{m}=\{g \in G \mid m g=m\} .
$$

Notice that even effective actions often have nontrivial stabilizers.

Proposition 2.0.11. If $G$ acts on $M$ then

$$
G / G_{m} \simeq m \cdot G
$$

as sets.

EXAmple 2.0.12. Let $M=P$ be the set of all lines in $\mathbb{R}^{3}$ containing the origin. Then the group of all linear automorphisms of $\mathbb{R}^{3}, G=\mathrm{GL}_{3}(\mathbb{R})$ acts on $M$. Let $m \in M$ be the $x$ axis. Then it is not hard to see that $G_{m}=\mathrm{GL}_{2}(\mathbb{R})$ and therefore

$$
P=\mathrm{GL}_{3}(\mathbb{R}) / \mathrm{GL}_{2}(\mathbb{R})
$$

We write

$$
p: M \rightarrow M / G
$$

for the mapping

$$
m \mapsto[m]
$$

If $M$ is a topological space and $G$ acts on $M$ then we can put a natural topology on $M / G$, namely a subset $U$ of $M / G$ is declared to be open if and only if $p^{-1}(U)$ is open in $M$.

EXAMPLE 2.0.13. $\tilde{X} / \pi_{1}(X) \simeq X$.

There are quotients in the category of sets, and also in the category of topological spaces.

But the category of smooth manifolds is quite unlike the category of sets or of topological spaces (for manifolds have structure sheafs).

\section{Orbifolds.}

Let $M=T^{2}=S^{1} \times S^{1}$ be a two-dimensional torus, and let $G=\mathbb{Z}_{2}$ be the finite subgroup of diffeomorphisms of $M$ given by the action

$$
(z, w) \mapsto(\bar{z}, \bar{w})
$$

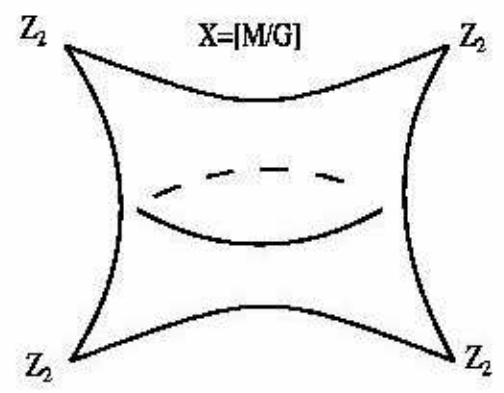

Pillowcase Orbifold 
ExAmPLE 3.0.14. Show that while the quotient space $X=M / G$ is topologically a sphere it is impossible to put a smooth structure on $X$ so that the quotient map $M \longrightarrow X$ will become smooth. It is in this sense that we say that $X$ is not a smooth manifold.

What will enlarge the category of smooth manifolds to a bigger category is called the category of orbifolds. Once we do this, when we consider the orbifolds M and $\mathrm{X}$ then the natural orbifold morphism $\mathrm{M} \longrightarrow \mathrm{X}$ becomes smooth.

While the orbifold $\mathrm{M}$ contains exactly the same amount of information as $M$ the orbifold $\mathrm{X}=[M / G]$ (known as a pillowcase) contains more information that the quotient space $X=M / G$. For instance $\mathrm{X}$ remembers that the action had 4 fixed points each with stabilizer $G$. It remembers in fact the stabilizer of every point, and how these stabilizers fit together. On the other hand $X$ does not remember neither the manifold $M$ nor the group $G$. In fact if we define $N$ to be two disjoint copies of $M$ and $H=G \times G$ to act on $M$ by letting $G \times 1$ act by complex conjugation on both copies as before, and $1 \times G$ act by swapping the copies then

$$
\mathrm{X}=[M / G] \cong[N / H] .
$$

Not every orbifold can be obtained from a finite group acting on a manifold. An orbifold is always locally the quotient of a manifold by a finite group but this may fail globally.

For example consider the teardrop:

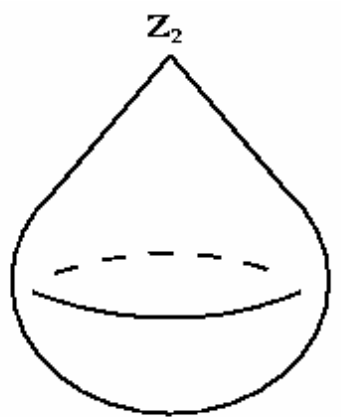

A Teardrop Orbifold

This orbifold may be obtained by gluing two global quotients. Consider the orbifold $\mathrm{X}_{1}=\left[\mathbb{C} / \mathbb{Z}_{2}\right]$ where $\mathbb{Z}_{2}$ acts by the holomorphic automorphism $z \mapsto-z$. Let $\mathrm{X}_{2}=\mathbb{C}$ simply be the complex line. Then we have in the category of orbifolds a diagram of inclusions

$$
\mathrm{X}_{1} \longleftarrow \mathbb{C}^{*} \longrightarrow \mathrm{X}_{2}
$$

and therefore we can glue $\mathrm{X}_{1}$ and $\mathrm{X}_{2}$ along $\mathbb{C}^{*}$ to obtaining the teardrop $\mathrm{X}$.

ExAmPLE 3.0.15. Prove that it is not possible to obtain the teardrop as a global quotient by a finite group.

There are several definitions of the concept of an orbifold. The first one due to Satake 25] was written using the so-called orbifold atlases, unfortunately quite a few concepts are a bit cumbersome using this definition. We opt to think of 
an orbifold as a certain kind of category following Grothendieck, Haefliger and Moerdijk [20].

For this reason we will start reviewing the basic facts about category theory. Category theory was discover by Eilenberg and MacLane in the 50's 19 and ever since has pervaded all fields of mathematics.

You may want to think of the category of sets as you read the following definition. The objects of the category of sets are all sets and the arrows are all mappings between them. You may also want to think of an object as a sort of dot and an arrow as something with a direction joining the dots.

Definition 3.0.16. A category consists of:

- A class $\mathcal{O} b j(\mathcal{C})$, that we will denote by $\mathcal{C}_{0}$, of objects of $\mathcal{C}$.

- A class $\operatorname{Arr}(\mathcal{C})$, that we will denote by $\mathcal{C}_{1}$, of arrows of $\mathcal{C}$. For each pair of objects $a$ and $b$ the class of all arrows from $a$ to $b$ is denoted by $\mathcal{C}(a, b)$.

- Two assignments $s_{\mathcal{C}}, t_{\mathcal{C}}: \mathcal{A r r}(\mathcal{C}) \rightarrow \mathcal{O} b j(\mathcal{C})$ called source and target respectively.

- Unit. An assignment $u_{\mathcal{C}}: \mathcal{O} b j(\mathcal{C}) \rightarrow \mathcal{A r r}(\mathcal{C})$ such that:

$$
s_{\mathcal{C}}\left(u_{\mathcal{C}}(a)\right)=t_{\mathcal{C}}\left(u_{\mathcal{C}}(a)\right)=a,
$$

for every $a \in \mathcal{O} b j(\mathcal{C})$.

- Composition Law. For each triple $a, b$ and $c$ of objects of $\mathcal{C}$ an assignment $m_{(a, b, c)}: \mathcal{C}(a, b) \times \mathcal{C}(b, c) \rightarrow \mathcal{C}(a, c)$, where its image on $(\alpha, \beta) \in \mathcal{C}(a, b) \times$ $\mathcal{C}(b, c)$ well be denoted by $\beta \circ \alpha$, satisfying the following properties:

(1) For every $a \in \mathcal{O} b j(\mathcal{C})$

$$
\begin{gathered}
s_{\mathcal{C}}\left(u_{\mathcal{C}}(a)\right)=t_{\mathcal{C}}\left(u_{\mathcal{C}}(a)\right)=a, \\
\mathcal{O} b j(\mathcal{C}) \stackrel{u}{\longrightarrow} \mathcal{A r r}(\mathcal{C}) \\
\left.\operatorname{Arr}(\mathcal{C}) \stackrel{t}{\longrightarrow} \mathcal{O}\right|_{s} \text { bj }(\mathcal{C})
\end{gathered}
$$

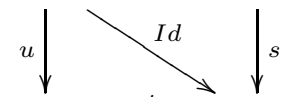

in other words the source and target of $u_{\mathcal{C}}(a)=a$ for every $a$.

(2) Associativity. For all $\alpha, \beta, \gamma \in \mathcal{A r r}(\mathcal{C})$ it holds that $\alpha \circ(\beta \circ \gamma)=$ $(\alpha \circ \beta) \circ \gamma$, formally for every elements $a, b, c, d$ fixed in $\mathcal{O} b j(\mathcal{C})$ we have

$$
\begin{gathered}
m_{(a, c, d)} \circ\left(m_{(a, b, c)} \times I d_{\mathcal{C}(c, d)}\right)=m_{(a, b, d)}\left(I d_{\mathcal{C}(a, b)} \times m_{(b, c, d)}\right), \\
\mathcal{C}(a, b) \times \mathcal{C}(b, c) \times \mathcal{C}(c, d) \stackrel{m_{a, b, c} \times I d_{\mathcal{C}(c, d)}}{\longrightarrow} \mathcal{C}(a, c) \times \mathcal{C}(c, d) \\
I d_{\mathcal{C}(a, b) \times m_{b, c, d}} \downarrow \\
\mathcal{C}(a, b) \times \mathcal{C}(b, d) \stackrel{\left.\right|_{(a, c, d)}}{\longrightarrow} \mathcal{C}(a, d)
\end{gathered}
$$

(3) Unity. For every $a, b \in \mathcal{O} b j(\mathcal{C})$ and $\alpha \in \mathcal{C}(a, b) \alpha=u_{\mathcal{C}}(b) \circ \alpha=$ $\alpha \circ u_{\mathcal{C}}(a)$ holds, formally

$$
m_{(a, b, b)}\left(\alpha, u_{\mathcal{C}}(b)\right)=m_{(a, a, b)}\left(u_{\mathcal{C}}(a), \alpha\right)=\alpha .
$$


ExAmple 3.0.17. Let us define Sets the category with objects the class of all spaces (proper class) and arrows the class of function of sets. The unity of this category assigns to each set $X$ the usual identity function of sets over $X$ and the function $m_{\mathcal{C}}$ the composition of functions, when it is defined.

ExAmple 3.0.18. The category Ab the subclass of Sets whose objects are all abelian groups and arrows the class of morphism of groups with the same unity and rule of composition as Sets. In he same manner are defined the categories Mod, Ring, Groups, etc.

ExAmple 3.0.19. The category Top of topological spaces and continuous functions.

ExAmple 3.0.20. Let us consider the category Corr of correspondences 8 whose objects are topological spaces and whose arrows (from $X$ to $Y$ ) are diagrams of continuous mappings of the form

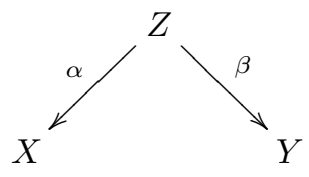

for $Z$ some topological space. We define the composition of arrows by

$$
(X \stackrel{\alpha}{\leftarrow} V \stackrel{\beta}{\rightarrow} Y) \circ(Y \stackrel{\gamma}{\leftarrow} W \stackrel{\delta}{\rightarrow} Z)=X \stackrel{\alpha}{\leftarrow} U \stackrel{\delta}{\rightarrow} Z
$$

where $U$ is defined as the fiber product

$$
U=V \times_{Y} W=\{(v, w) \mid \beta(v)=\gamma(w)\} .
$$

Observe that the ordinary category of topological spaces can be embedded as a subcategory of Corr since a continuous map $f: X \rightarrow Y$ can be interpreted as the correspondence

$$
X \stackrel{\pi_{X}}{\longleftarrow} \mathcal{G}_{f} \stackrel{\pi_{Y}}{\longrightarrow} Y,
$$

where $\mathcal{G}_{f}=\{(x, y) \mid y=f(x)\}$ is the graph of $f$. This is functorial for we have

$$
\mathcal{G}_{f} \times_{Y} \mathcal{G}_{h}=\mathcal{G}_{h \circ f} .
$$

Unfortunately homology is not a functor from Corr to graded abelian groups. Nevertheless suppose that we have a correspondence $X \stackrel{\alpha}{\leftarrow} Z \stackrel{\beta}{\rightarrow} Y$ where

- $X, Y$ and $Z$ are manifolds (possibly infinite dimensional).

- $\alpha$ is a regular embedding of finite codimension $d$.

In this case we say that $X \stackrel{\alpha}{\longleftarrow} Z \stackrel{\beta}{\rightarrow} Y$ is a smooth correspondence of degree $-d$. In any case using the Gysin map we can produce the composition

$$
H_{*}(X) \stackrel{\alpha_{!}}{\rightarrow} H_{*-d}(Z) \stackrel{\beta_{*}}{\rightarrow} H_{*-d}(Y)
$$

which is the induced homomorphism of degree $-d$ in homology.

DEFINITION 3.0.21. A Groupoid is a category in which each arrow has an inverse, namely for each pair $a, b \in \mathcal{O} b j \mathcal{C}$ and each $\alpha \in \mathcal{C}(a, b)$ there exist an arrow $\alpha^{-1} \in \mathcal{C}(b, a)$ in such a way that $\alpha^{-1} \circ \alpha=u(a)$ y $\alpha \circ \alpha^{-1}=u(b)$. In this case we will denote by $i: \mathcal{C}(a, b) \rightarrow \mathcal{C}(b, a)$ the map that assigns to each arrows its inverse. 
EXAMPLE 3.0.22. Let $G$ be a group acting on a set $M$. Let $G \ltimes M$ be the groupoid whose objects are the set $\mathrm{M}$, and arrows $g: x \rightarrow y$ such that $y=g x$, this set can be seen as the set $G \times M$. Here the composition is defined of natural manner $g g^{\prime}: x \rightarrow z$ where $g^{\prime}: x \rightarrow y$ and $g: y \rightarrow z$. For each object $x$ the unit map associates the unit $e$ of $G$. The structure maps are defined in the obvious way as $s: G \times M \rightarrow M$ the projection and $t: G \times M \rightarrow M$ the action.

EXAMPLE 3.0.23. Smooth manifolds provide a natural source of groupoids. Let $M$ be a smooth manifold. It is well known that a smooth manifold is a pair $(M, \mathcal{U})$ of a (Hausdorff, paracompact) topological space $M$ together with an atlas $\mathcal{U}=\left(U_{i}\right)_{i \in I}$, and is only by abuse of notation that we speak of a manifold $M$. In fact a smooth manifold is actually an equivalence class of a pair $[M, \mathcal{U}]$ where we say that $\left(M, \mathcal{U}_{1}\right) \sim\left(M, \mathcal{U}_{2}\right)$ if and only if there is a common refinement $\left(M, \mathcal{U}_{3}\right)$ of the atlas. We can say this in a slightly different way that will be easier to generalize to the case of orbifolds. To have a pair $(M, \mathcal{U})$ is the same thing as to have a small topological category $\mathrm{M}_{\mathcal{U}}$ defined as follows.

- Objects: Pairs $(m, i)$ so that $m \in U_{i}$. We endow the space of objects with the topology

$$
\coprod_{i} U_{i}
$$

- Arrows: Triples $(m, i, j)$ so that $m \in U_{i} \cap U_{j}=U_{i j}$. An arrow acts according to the following diagram.

$$
(x, i) \stackrel{(x, i, j)}{\longrightarrow}(x, j) .
$$

- The composition of arrows is given by

$$
(x, i, j) \circ(x, j, k)=(x, i, k)
$$

The topology of the space of arrows in this case is

$$
\coprod_{(i, j)} U_{i j}
$$

The category $\mathrm{M}$ is actually a groupoid, in fact

$$
(x, i, j) \circ(x, j, i)=(x, i, i)=I d_{(x, i)} .
$$

We will therefore define a manifold to be the equivalence class of the groupoid $\mathrm{M}_{\mathcal{U}}$ by an equivalence relation called Morita equivalence (that will amount exactly to the equivalence of atlases in this case).

3.1. Homotopies. The category Top of topological spaces with continuous mappings has an interesting additional structure. Homotopies of smooth mappings. This endows $\operatorname{Top}(X, Y)$ with the structure of a category. We will call a category with this additional structure a bicategory.

The category Cat of all categories is also a bicategory. Let us define the homotopies between functors. Let $F$ and $D$ functors from $\mathcal{C}$ to $\mathcal{B}$, a homotopy of functors is a functor $H: \mathcal{C} \times \mathcal{I} \rightarrow \mathcal{B}$ where $\mathcal{I}$ is a category with two objects and one arrow going between them, and the restrictions of $H$ to the two copies of $\mathcal{C}$ above, coincide with $F$ and $D$ respectively. The reader can verify that to have a homotopy between functors is the same as having a natural transformation.

Definition 3.1.1. A natural transformation of functors is a map $\Phi: \mathcal{C}_{0} \rightarrow \mathcal{B}_{1}$ in such a way that 
- For every $a \in \mathcal{C}_{0}, \Phi(a) \in \mathcal{B}(F(a), D(a))$, and

- For each $\alpha \in \mathcal{C}(a, b)$

$$
\begin{gathered}
\Phi(b) \circ F(\alpha)=D(\alpha) \circ \Phi(a) \\
F(a) \stackrel{F(\alpha)}{\longrightarrow} F(b) \\
\Phi(a) \downarrow \\
D(a) \stackrel{D(\alpha)}{\longrightarrow} D(b)
\end{gathered}
$$

\section{Groupoids}

DeFinition 4.0.2. A Lie groupoid $G$ is a category in which every morphism is invertible such that $G_{0}$ and $G_{1}$, the sets of objects and morphism respectively, are smooth manifolds. We will denote the structure maps by:

$$
\mathrm{G}_{1}{ }_{t}{ }_{s} \mathrm{G}_{1} \stackrel{m}{\longrightarrow} \mathrm{G}_{1} \stackrel{i}{\longrightarrow} \mathrm{G}_{1} \stackrel{s}{\underset{t}{\longrightarrow}} \mathrm{G}_{0} \stackrel{e}{\longrightarrow} \mathrm{G}_{1}
$$

where $s$ and $t$ are the source and the target maps, $m$ is the composition (we can compose two arrows whenever the target of the first equals the source of the second), $i$ gives us the inverse arrow, and $e$ assigns the identity arrow to every object. We will assume that all the structure maps are smooth maps. We also require the maps $s$ and $t$ to be submersions, so that $\mathrm{G}_{1 t} \times{ }_{s} \mathrm{G}_{1}$ is also a manifold.

Definition 4.0.3. The stabilizer $\mathrm{G}_{x}$ of a groupoid $\mathrm{G}$ on $x \in \mathrm{G}_{0}$ is the set of arrows whose source and target are both $x$. Notice that $\mathrm{G}_{x}$ is a group

Definition 4.0.4. A topological (Lie) groupoid is called étale if the source and target maps $s$ and $t$ are local homeomorphisms (local diffeomorphisms).

For an étale groupoid we will mean a topological étale groupoid.

We will always denote groupoids by letters of the type G, H, S.

We will also assume that the anchor map $(s, t): \mathrm{G}_{1} \rightarrow \mathrm{G}_{0} \times \mathrm{G}_{0}$ is proper, groupoids with this property are called proper groupoids. This will force all stabilizers to be finite.

Definition 4.0.5. A morphism of groupoids $\Psi: \mathrm{H} \rightarrow \mathrm{G}$ is a pair of maps $\Psi_{i}: \mathrm{H}_{i} \rightarrow \mathrm{G}_{i} i=0,1$ such that they commute with the structure maps. The maps $\Psi_{i}$ will be required to be smooth.

The morphism $\Psi$ is called Morita if the following square is a cartesian square .

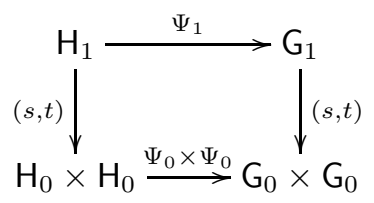

and if $s \circ \pi_{2}: \mathrm{H}_{0} \Psi_{0} \times{ }_{t} \mathrm{G}_{1} \rightarrow \mathrm{G}_{0}$ is an open surjection.

Two groupoids $G$ and $H$ are Morita equivalent if there exist another groupoid $\mathrm{K}$ with Morita morphisms $\mathrm{G} \stackrel{\simeq}{\longleftarrow} \mathrm{K} \stackrel{\simeq}{\rightrightarrows} \mathrm{H}$.

A theorem of Moerdijk [20 states that the category of orbifolds is equivalent to a quotient category of the category of proper étale groupoids after formally inverting the Morita morphisms. 
Whenever we write orbifold, we will choose a proper étale smooth groupoid representing it (up to Morita equivalence.)

Example 4.0.6. Consider again the pillowcase (as in example 3.0.14). Define the following groupoids.

- The groupoid $\mathrm{G}$ whose space of objects are elements $m \in M$ with the topology of $M$, and whose space of arrows is the set of pairs $(m, g)$ with the topology of $M \times G$. We have the diagrams

$$
m \stackrel{(m, g)}{\rightarrow} m g
$$

and the composition law

$$
(m, g) \circ(m g, h)=(m, g h) .
$$

- Similarly we define the groupoid $\mathrm{H}$ using the action of $H$ in $N$ with objects $n \in N$ and arrows $(n, h) \in N \times H$.

The orbifold $X$ is the equivalence class of the groupoid $G$. Since there is a Morita morphism $H \rightarrow G$, we can say also that $X$ is the equivalence class of $H$. By abuse of notation we will often say that $\mathrm{G}$ is an orbifold when we really mean that its equivalence class is the orbifold.

EXAMPLE 4.0.7. More generally, let $M$ be a smooth manifold and $G \subset \operatorname{Diff}(M)$ be a finite group acting on it.

- We say that the orbifold $[M / G]$ is the equivalence class of the groupoid $\mathrm{X}$ with objects $m \in M$ and arrows $(m, g) \in M \times G$.

- We can define another groupoid representing the same orbifold as follows. Take a contractible open cover $\mathcal{U}=\left\{U_{i}\right\}_{i \in I}$ of $M$ such that all the finite intersections of the cover are either contractible or empty, and with the property that for any $g \in G$ and any $i \in I$ there exists $j \in I$ so that $U_{i} g=U_{j}$. Define $\mathrm{G}_{0}$ as the disjoint union of the $U_{i}$ 's with $\mathrm{G}_{0} \stackrel{\rho}{\rightarrow} M=\mathrm{X}_{0}$ the natural map. Take $G_{1}$ as the pullback square

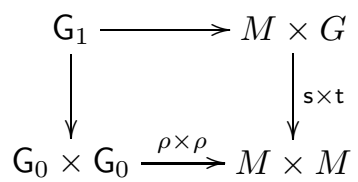

where $\mathbf{s}(m, g)=m$ and $\mathrm{t}(m, g)=m g$. From the construction of $\mathrm{G}$ we see that we can think of $G_{1}$ as the disjoint union of all the intersections of two sets on the base times the group $G$, i.e.

$$
\mathrm{G}_{1}=\left(\bigsqcup_{(i, j) \in I \times I} U_{i} \cap U_{j}\right) \times G
$$

where the arrows in $U_{i} \cap U_{j} \times\{g\}$ start in $\left.U_{i}\right|_{U_{j}}$ and end in $\left(\left.U_{j}\right|_{U_{i}}\right) g$. This defines a proper étale Leray groupoid $G$ and by definition it is Morita equivalent to $\mathrm{X}$. 


\section{Moduli Spaces}

Moduli spaces are often given by orbifolds. Moduli spaces are "spaces" that contain the universal family of objects of certain kind. If $X$ is the moduli space of objects of certain kind we want

$$
\operatorname{Maps}(S, \mathrm{X})
$$

to classify families of objects of this kind over $S$. This is akin to the situation in topology in which we represent, for example $n$-dimensional vector bundles over $M$ up to isomorphism by homotopy classes of maps to a certain universal space $B \mathrm{U}(n)$. Remember that $B \mathrm{U}(n)=\mathrm{Gr}_{n}\left(\mathbb{C}^{\infty}\right)$. Moduli spaces are often not spaces at all but rather orbifolds.

EXAMPLE 5.0.8. Let us consider the moduli space of triangles $\mathcal{T}$. We identify an Euclidean triangle $T$ with a triple

$$
T=(a, b, c)
$$

satisfying the triangle inequalities

$$
\begin{aligned}
& a+b>c, \\
& b+c>a,
\end{aligned}
$$

and

$$
c+a>b .
$$

The set $M$ of all such $T$ is diffeormorphic to

$$
M \approx \Delta \times \mathbb{R}^{+} .
$$

It is a positive cone over an equilateral triangle (of triangles of fixed perimeter $a+b+c)$ that we denote by $\Delta$.

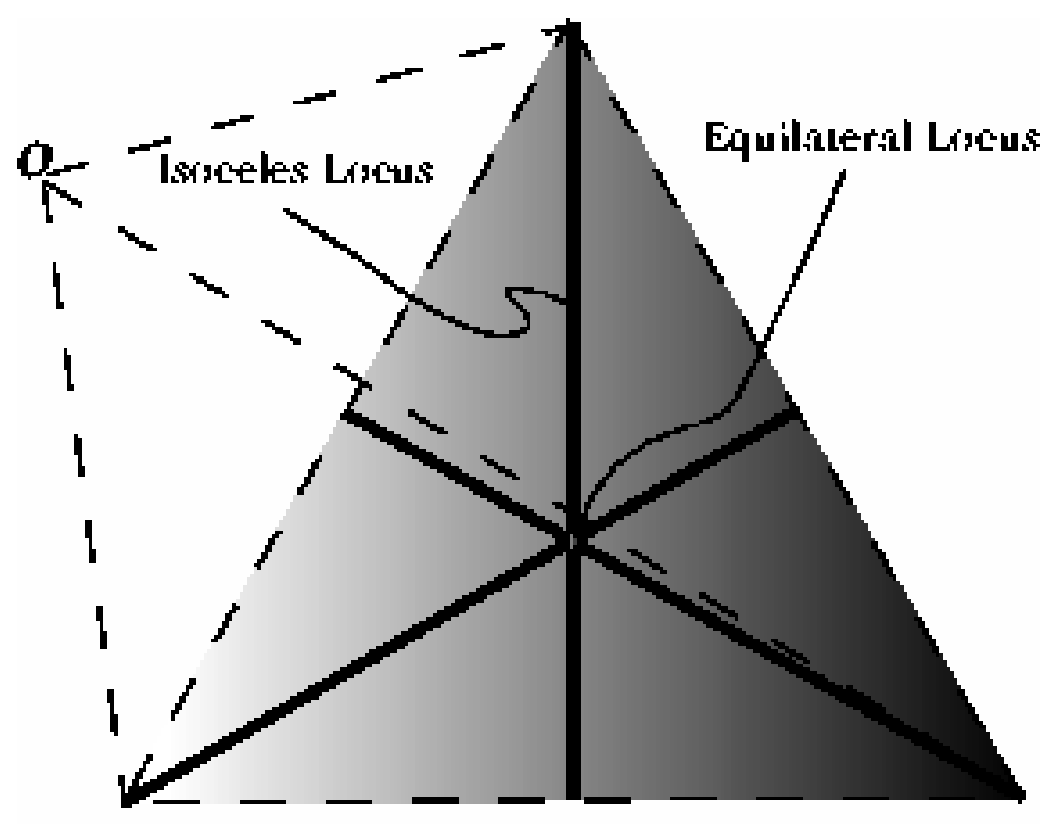

The Moduli space of Triangles 
The is a natural action of $\mathfrak{S}_{3}$ on $M$ by multiplication of the corresponding permutation matrix. The moduli orbifold of triangles is

$$
\mathcal{T}=\left[M / \mathfrak{S}_{3}\right]
$$

Now the class of smooth families of triangles over the circle $S=S^{1}$ is now endowed naturally with the structure of an orbifold:

$$
\mathcal{T}^{S}=\left[P / \mathfrak{S}_{3}\right]
$$

where $P$ is the family of paths $I=[0,1] \rightarrow M$ so that

$$
\gamma(1)=\gamma(0) \cdot g
$$

for some $g \in \mathfrak{S}_{3}$. This is what we have called the loop orbifold 16, 17. We will come back to this later.

\section{Almost Free Lie Group Actions.}

We will suppose now that $K$ is a Lie group. Let $M$ be a smooth manifold in which $K$ is acting. We say that $M$ is a $K$-manifold.

A map $\phi: M \rightarrow N$ between $K$-manifolds is said to be equivariant if

$$
\phi(x g)=\phi(x) g .
$$

We say that a vector bundle $E \longrightarrow M$ is a $K$-vector bundle if $K$ acts linearly on the fibers and the projection map is equivariant.

Stabilizers $K_{m}$ of Lie group actions are closed subgroups and hence Lie groups. Stabilizers of points in the same orbit are conjugate to each other:

$$
K_{m g}=g^{-1} K_{m} g
$$

The conjugacy class of a subgroup $H$ will be written $(H)$. Hence $\left(K_{m}\right)$ only depends on the orbit of $m$ and not on $m$. Given $m \in M$ he map

$$
f_{m}: K / K_{m} \longrightarrow M
$$

given by

$$
f_{m}(\bar{g})=m g,
$$

is an injective immersion. It does not follow that $m \cdot K \subseteq M$ is a submanifold. Just think of a torus with an irrational flow. Nonetheless, of course, if $K$ is compact then $m \cdot K \subseteq M$ is always a submanifold. If $K$ is compact $M / K$ is Hausdorff and $p: M \rightarrow M / K$ is proper and closed. So, from now on we shall suppose that $K$ is compact. Fix $m \in M$ and let

$$
V_{m}=T_{m} M / T_{m}(m K) .
$$

Notice that for $g \in K_{m}$ we have

$$
d_{m} g: T_{m} M \longrightarrow T_{m g} M=T_{m} M
$$

Therefore

$$
K_{m} \longrightarrow \operatorname{Aut}\left(V_{m}\right) \text {. }
$$

Also $K_{m}$ acts freely on $K \times V_{m}$, by $h(g, v)=\left(g h^{-1}, h v\right)$. This defines a vector bundle $K \times_{K_{m}} V_{m} \longrightarrow K / K_{m}$.

Theorem 6.0.9 (The Slice Theorem (Koszul 1953) [14]). There exists an equivariant diffeomorphism from an equivariant open neighborhood of the zero section of $K \times_{K_{m}} V_{m} \longrightarrow K / K_{m}$ to an open neighborhood of $m K \subseteq M$, sending the zero section to $m K$ by $f_{m}$. 
The union of all the orbits of a given type is a submanifold of $M$. If $M$ is compact there are only finitely many orbit types.

From now on we will suppose that all $K_{m}$ are finite, and that $M / K$ is connected. Then there exists a finite group $G$ so that the set of points in $M$ with stabilizers conjugate to $G$ (denoted by $M_{(G)}$ ) is open and dense in $M$. (Prove it by induction over the dimension of the manifold $M$, and consider the sphere bundle of the neighborhoods of the theorem.)

If $K$ is a compact Lie group acting on $M$, and each stabilizer $K_{x}$ is finite, then $K \ltimes M$ is an orbifold groupoid. Observe that the slice theorem for compact group actions gives for each point $x$ a "slice" $V_{x} \subseteq M$ for which the action defines a diffeomorphism $K \times_{K_{x}} V_{x} \hookrightarrow M$ onto a saturated open neighborhood $U_{x}$ of $x$. Then $K_{x} \ltimes V_{x}$ is an étale groupoid which is Morita equivalent to $K \ltimes U_{x}$. Patching these étale groupoids together for sufficiently many slices $V_{x}$ yields an étale groupoid Morita equivalent to $K \ltimes M$ [2].

Definition 6.0.10. A orbivector bundle over $\mathbf{X}$ is a pair $(E, \theta)$ where $E$ is an ordinary vector bundle over $\mathrm{X}_{0}$ and $\theta$ is an isomorphism $s^{*} E \cong t^{*} E$ (here we are choosing a representative of the Morita class)

EXAmple 6.0.11. This recovers the usual definition for a manifold acted on by the identity group.

EXAMPLE 6.0.12. For the groupoid $G \ltimes M$ this gives the usual definition of an equivariant vector bundle. The tangent bundle $T \mathrm{X}$ of an orbifold $\mathrm{X}$ is a orbibundle over X.

EXAMPLE 6.0.13. If $U=[V / G]$ is a local chart (namely the restriction of the groupoid to a very small neighborhood), then a corresponding local uniformizing system for $T \mathrm{X}$ will be $[T V / G]$ with the action $g \cdot(x, v)=\left(g x, d g_{x}(v)\right)$.

Definition 6.0.14. Given an orbifold $\mathrm{X}$ we say that the space $X=\mathrm{X}_{1} / \sim$ is its coarse topological space, or quotient space. Here $x \sim y$ whenever there is an arrow from $x$ to $y$. We will often write $\pi: \mathrm{X}_{0} \rightarrow X$ to denote the canonical projection.

Definition 6.0.15. Given a point $x \in X$ and an open neighborhood $x \in U \subseteq X$ we define $\mathrm{X}_{U}$ to be the restricted groupoid, namely its objects are $V=\pi^{-1} U$ and its arrows are all arrows $\alpha$ such that both $\pi(s(\alpha))$ and $\pi(t(\alpha))$ are in $U$. It is easy to show that for a sufficiently small $U$ we have that $\mathrm{X}_{U}$ is isomorphic to $[V / G]$ for some finite group $G$ acting on the manifold $V$. Such orbifold $[V / G]$ is called a local orbifold chart, or sometimes, a uniformizing system. An orbifold is called effective if at every point of $X$ we can find a local orbifold chart where the action of $G$ in $V$ is effective.

Similarly the frame bundle $P(\mathrm{X})$ is a principal orbibundle over $\mathrm{X}$. The local uniformizing system is $U \times O(n) / G$ with local action $g \cdot(x, A)=(g x, d g \circ A)$. Notice that if the orbifold is effective then $P(\mathrm{X})$ is always a smooth manifold for the local action is free and $(s, t): \mathrm{X}_{1} \rightarrow \mathrm{X}_{0} \times \mathrm{X}_{0}$ is one-to-one. From this we deduce that $\mathrm{X}=[P(\mathrm{X}) / O(n)]$. This proves the following proposition.

Proposition 6.0.16. Every effective orbifold arises from the almost free action of a Lie group on a manifold. 


\section{The Algebraic Topology of Orbifolds.}

Define

$$
\mathrm{X}^{(n)}:=\underbrace{\mathrm{X}_{1 t} \times_{s} \cdots{ }_{t} \times{ }_{s} \mathrm{X}_{1}}_{n}
$$

In the case in which $X_{1}$ is a set then $X^{(n)}$ is the set of sequences $\left(\gamma_{1}, \gamma_{2}, \ldots, \gamma_{n}\right)$ so that we can form the composition $\gamma_{1} \circ \gamma_{2} \circ \cdots \circ \gamma_{n}$.

With this data we can form a simplicial set [26].

Definition 7.0.17. A (semi-) simplicial set (resp. group, space, scheme) $X_{\bullet}$ is a sequence of sets $\left\{X_{n}\right\}_{n \in \mathbb{N}}$ (resp. groups, spaces, schemes) together with maps

$$
\begin{gathered}
\quad X_{0} \leftrightarrows X_{1} \leftrightarrows X_{2} \leftrightarrows \cdots \leftrightarrows X_{m} \leftrightarrows \cdots \\
\partial_{i}: X_{m} \rightarrow X_{m-1}, \quad s_{j}: X_{m} \rightarrow X_{m+1}, \quad 0 \leq i, j \leq m
\end{gathered}
$$

called boundary and degeneracy maps, satisfying

$$
\begin{aligned}
\partial_{i} \partial_{j} & =\partial_{j-1} \partial_{i} \quad \text { if } i<j \\
s_{i} s_{j} & =s_{j+1} s_{i} \text { if } i<j \\
\partial_{i} s_{j} & = \begin{cases}s_{j-1} \partial_{i} & \text { if } i<j \\
1 & \text { if } i=j, j+1 \\
s_{j} \partial_{i-1} & \text { if } i>j+1\end{cases}
\end{aligned}
$$

The nerve of a category (following Segal $\mathbf{2 6}$ ) is a semi-simplicial set $N \mathcal{C}$ where the objects of $\mathcal{C}$ are the vertices, the morphisms the 1-simplices, the triangular commutative diagrams the 2 -simplices, and so on.

For a category coming from a groupoid then the corresponding simplicial object will satisfy $N \mathcal{C}_{n}=X_{n}=\mathrm{X}^{(n)}$.

We can define the boundary maps $\partial_{i}: \mathbf{X}^{(n)} \rightarrow \mathbf{X}^{(n-1)}$ by:

$$
\partial_{i}\left(\gamma_{1}, \ldots, \gamma_{n}\right)= \begin{cases}\left(\gamma_{2}, \ldots, \gamma_{n}\right) & \text { if } i=0 \\ \left(\gamma_{1}, \ldots, m\left(\gamma_{i}, \gamma_{i+1}\right), \ldots, \gamma_{n}\right) & \text { if } 1 \leq i \leq n-1 \\ \left(\gamma_{1}, \ldots, \gamma_{n-1}\right) & \text { if } i=n\end{cases}
$$

and the degeneracy maps by

$$
s_{j}\left(\gamma_{1}, \ldots, \gamma_{n}\right)= \begin{cases}\left(e\left(s\left(\gamma_{1}\right)\right), \gamma_{1}, \ldots, \gamma_{n}\right) & \text { for } j=0 \\ \left(\gamma_{1}, \ldots, \gamma_{j}, e\left(t\left(\gamma_{j}\right)\right), \gamma_{j+1}, \ldots, \gamma_{n}\right) & \text { for } j \geq 1\end{cases}
$$

We will write $\Delta^{n}$ to denote the standard $n$-simplex in $\mathbb{R}^{n}$. Let $\delta_{i}: \Delta^{n-1} \rightarrow \Delta^{n}$ be the linear embedding of $\Delta^{n-1}$ into $\Delta^{n}$ as the $i$-th face, and let $\sigma_{j}: \Delta^{n+1} \rightarrow \Delta^{n}$ be the linear projection of $\Delta^{n+1}$ onto its $j$-th face.

Definition 7.0.18. The geometric realization $\left|X_{\bullet}\right|$ of the simplicial object $X_{\bullet}$ is the space

$$
\left|X_{\bullet}\right|=\left(\coprod_{n \in \mathbb{N}} \Delta^{n} \times X_{n}\right) / \begin{aligned}
\left(z, \partial_{i}(x)\right) & \sim\left(\delta_{i}(z), x\right) \\
\left(z, s_{j}(x)\right) & \sim\left(\sigma_{j}(z), x\right)
\end{aligned}
$$

Notice that the topologies of $X_{n}$ are relevant to this definition.

The simplicial object $N \mathcal{C}$ determines $\mathcal{C}$ and its topological realization is called $B \mathcal{C}$, the classifying space of the category. Again in our case $\mathcal{C}$ is a topological category in Segal's sense. 
Definition 7.0.19. For a groupoid $\mathrm{X}$ we will call $B \mathrm{X}=|N \mathrm{X}|$ the classifying space of the orbifold.

The following proposition establishes that $B$ is a functor from the category of groupoids to that of topological spaces. Recall that we say that two morphisms of groupoids are Morita related if the corresponding functors for the associated categories are connected by a morphism of functors.

Proposition 7.0.20. A morphism of groupoids $\mathrm{X}_{1} \rightarrow \mathrm{X}_{2}$ induces a continuous map $B \mathrm{X}_{1} \rightarrow B \mathrm{X}_{2}$. Two morphism that are Morita related will produce homotopic maps. In particular a Morita equivalence $\mathrm{X}_{1} \sim \mathrm{X}_{2}$ will induce a homotopy equivalence $B \mathrm{X}_{1} \simeq B \mathrm{X}_{2}$. This assignment is functorial.

EXAmPLE 7.0.21. For the groupoid $\bar{G}=(\star \times G \rightrightarrows \star)$ the space $B \bar{G}$ coincides with the classifying space $B G$ of $G$.

Consider now the groupoid $\mathrm{X}=(G \times G \rightrightarrows G)$ where $s\left(g_{1}, g_{2}\right)=g_{1}, t\left(g_{1}, g_{2}\right)=$ $g_{2}$ and $m\left(\left(g_{1}, g_{2}\right) ;\left(g_{2}, g_{3}\right)\right)=\left(g_{1}, g_{3}\right)$ then it is easy to see that $B \mathrm{X}$ is contractible and has a $G$ action. Usually $B \mathrm{X}$ is written $E G$

A morphism of groupoids $\mathrm{X} \rightarrow \bar{G}$ is the same thing as a principal $G$ bundle over $\mathrm{X}$ and therefore can be written by means of a map $G \times G \rightarrow G$. If we choose $\left(g_{2}, g_{2}\right) \mapsto g_{1}^{-1} g_{2}$ the induced map of classifying spaces

$$
E G \longrightarrow B G
$$

is the universal principal $G$-bundle fibration over $B G$.

EXAMPLE 7.0.22. Consider a smooth manifold $X$ and a good open cover $\mathcal{U}=$ $\left\{U_{\alpha}\right\}_{\alpha}$. Consider the groupoid $\mathcal{G}=\left(\mathcal{G} \rightrightarrows \mathcal{G}_{0}\right)$ where $\mathcal{G}_{1}$ consists on the disjoint union of the double intersections $U_{\alpha \beta}$. Segal calls $X_{U}$ the corresponding topological category. Then he proves that $B \mathcal{G}=B X_{U} \simeq X$.

If we are given a principal $G$ bundle over $\mathcal{G}$ then we have a morphism $\mathcal{G} \rightarrow \bar{G}$ of groupoids, that in turn induces a map $X \rightarrow B G$. Suppose that in the previous example we take $G=\mathrm{GL}_{n}(\mathbb{C})$. Then we get a map $X \rightarrow B \mathrm{GL}_{n}(\mathbb{C})=B U$.

EXAmple 7.0.23. Consider a groupoid $\mathrm{X}$ of the form $M \times G \rightrightarrows M$ where $G$ is acting on $M$ continuously. Then $B \mathrm{X} \simeq E G \times{ }_{G} M$ is the Borel construction for the action $M \times G \rightarrow M$.

Definition 7.0.24. The fundamental group of $\mathbf{X}$ is defined to be $\pi_{1}(\mathbf{X})=$ $\pi_{1}(B \mathrm{X})$. Similarly for the cohomology $H^{*}(\mathrm{X})=H^{*}(B \mathrm{X})$.

This last definition of cohomology is a bit too naive whenever we have obtained our orbifold by some geometric procedures. For example, as the space of solutions of algebraic equations. We will return to this issue later once we have the perspective given to us by topological quantum field theories.

\section{Loop Orbifolds}

The loop space is slightly more complicated in the case of an orbifold.

To generalize this situation to an orbifold $\mathrm{X}$ (replacing the rôle of $M$ above), we must be able to say what is the candidate to replace $\mathcal{L} M$. This was done for a general orbifold in [16. The basic idea is that to a groupoid $X$ we must assign a new (infinite-dimensional) groupoid LX that takes the place of the free loopspace of $M$ in a functorial manner

\section{L: Orbifolds $\rightarrow$ Orbifolds.}


In 17 we prove the following.

TheOREM 8.0.25. The functor $\mathrm{L}$ defined in 16 commutes with the functor $B$ from groupoids to spaces defined in the previous section. Namely there is an homotopy equivalence

$$
B \mathrm{LX} \simeq \mathcal{L} B \mathrm{X}
$$

In the case in which $\mathrm{X}=[M / G]$, we proved that $\mathrm{LX}$ admits a much smaller and very concrete model defined as follows. The objects of the loop groupoid are given by

$$
(\mathrm{LX})_{0}:=\bigsqcup_{g \in G} \mathcal{P}_{g}
$$

where $\mathcal{P}_{g}$ is the set of all pairs $(\gamma, g)$ with $\gamma: \mathbb{R} \rightarrow X$ and $g \in G$ with $\gamma(t) g=$ $\gamma(2 \pi+t)$. The space of arrows of the loop groupoid is

$$
(\mathrm{LX})_{1}:=\bigsqcup_{g \in G} \mathcal{P}_{g} \times G
$$

and the action of $G$ in $\mathcal{P}_{g}$ is by translation in the first coordinate and conjugation in the second; that is, a typical arrow in the loop groupoid looks like

$$
(\gamma, g) \stackrel{((\gamma, g) ; h)}{\longrightarrow}\left(\gamma \cdot h, h^{-1} g h\right),
$$

or pictorially:

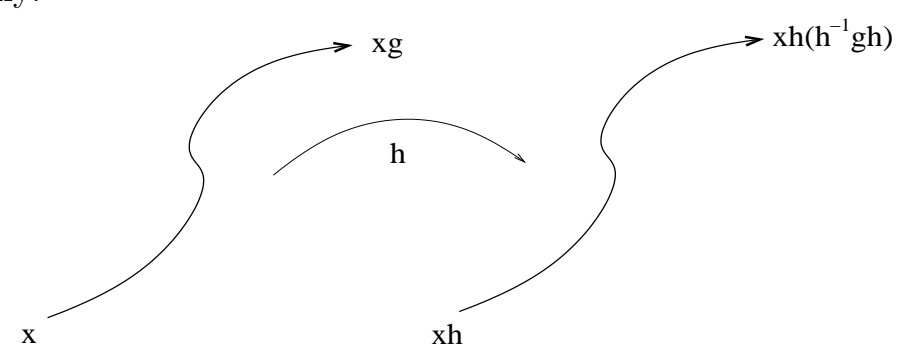

Theorem 8.0.26 (The Localization Principle [9]). Let $\mathrm{X}$ be an orbifold and $\mathbf{L X}$ its loop orbifold. Then the fixed orbifold under the natural circle action by rotation of loops is

$$
(\mathrm{LX})^{S^{1}}=I(\mathrm{X})
$$

where the groupoid $I(\mathrm{X})$ has as its space of objects

$$
I(\mathrm{X})_{0}=\left\{\alpha \in \mathrm{X}_{1}: s(\alpha)=t(\alpha)\right\}=\coprod_{m \in \mathrm{X}_{0}} \operatorname{AutX}(m)
$$

and its space of arrows is

$$
I(\mathrm{X})_{1}=Z\left(I\left(\mathrm{X}_{0}\right)\right)=\left\{g \in \mathrm{X}_{1}: \alpha \in I(\mathrm{X})_{0} \Rightarrow g^{-1} \alpha g \in I(\mathrm{X})_{0}\right\},
$$

a typical arrow in $I(\mathrm{X})$ from $\alpha_{0}$ to $\alpha_{1}$ looks like

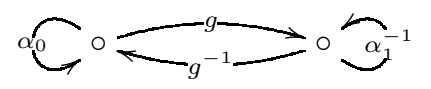

While for a smooth manifold the space of constant maps is

$$
M=(\mathcal{L} M)^{S^{1}}
$$

we have in contrast

$$
\mathrm{X} \subset I(\mathrm{X})=(\mathrm{LX})^{S^{1}}
$$


In 18 we define the ghost loop space $\mathcal{L}_{s} B \mathrm{X}$ as the subspace of elements $\gamma \in$ $\mathcal{L} B \mathrm{X}$ so that the composition with the canonical projection $\pi_{\mathrm{X}}: B \mathrm{X} \rightarrow X, \pi_{\mathrm{X}} \circ \gamma$ is constant. In that paper it is proved the homotopy equivalence

$$
B I(\mathrm{X}) \simeq \mathcal{L}_{s} B \mathrm{X}
$$

\section{Orbifold TQFTs}

9.1. Finite Groups. There is a very beautiful example of a topological quantum field theory (TQFT) due to Dijkgraaf and Witten $\mathbf{1 0}, \mathbf{2 7}, \mathbf{8}$. This is a $(n+1)$ dimensional TQFT $\left(H^{G}, \Psi^{G}, Z^{G}\right)_{n+1}$ associated to a finite group $G$. In this model we have

- $\mathcal{F}(Y)=[Y, B G]=\operatorname{Bun}_{G}(Y)$, where $\operatorname{Bun}_{G}(Y)$ is the set isomorphism classes of $G$-principal bundles on $Y$.

- $H^{G}(Y)=\operatorname{Map}\left(\operatorname{Bun}_{G}(Y), \mathbb{C}\right)$. Here we remark that $\operatorname{Bun}_{G}(Y) \cong \operatorname{Hom}_{\mathbb{Z}}\left(\pi_{1}(Y), G\right) / \sim$, this last bijection being induced by the holonomy of the bundle. The symbol $\sim$ denotes conjugation.

- For a boundaryless $Y$ we have $Z^{G}(Y)=\left|\operatorname{Hom}\left(\pi_{1}(Y) ; G\right)\right| /|G|$.

- If $\partial Y=Z$ has no output boundary then for each $P \in \operatorname{Bun}_{G}(Z)$ we have

$$
\Psi_{Y}(P)=\sum_{Q \in \operatorname{Bun}_{G}(Y), Q \mid Z=P} \frac{1}{|\operatorname{Aut}(Q)|} \in \mathbb{C}
$$

Segal has shown that when de dimension of the model is $1+1$ then we have

- The Frobenius algebra $\left(A_{G}, \theta_{G}\right)$ associated to $\left(H^{G}, \Psi^{G}, Z^{G}\right)_{1+1}$ is isomorphic to the center of the group algebra $\mathbb{C}[G]$, with trace

$$
\theta_{G}\left(\sum_{g} \lambda_{g} g\right)=\frac{1}{|G|} \lambda_{1}
$$

- For a boundaryless Riemann surface $\Sigma$ we have

$$
Z(\Sigma)=|G|^{2 g-2} \sum_{V} \frac{1}{(\operatorname{dim} V)^{2 g-2}}
$$

where $g$ is the genus of $\Sigma$ and $V$ runs through irreducible representations of $G$.

9.2. Orbifold String Topology. Orbifold string topology 17 is a quantum field theory that generalizes simultaneously the models of Dijkgraaf-Witten and of Chas-Sullivan described above, actually the theory interpolates between those two theories.

We fix a compact oriented orbifold $X$. This is a (positive boundary) $(1+1)$ dimensional topological quantum field theory with fields

$$
\mathcal{F}(Y)=\operatorname{Map}(Y, \mathbf{X})
$$

the moduli space of all orbifold morphisms from $Y$ to $\mathrm{X}$. We have replaced the manifold $M$ of string topology with an orbifold $\mathrm{X}$. The Dijkgraaf-Witten theory is recovered when we consider the orbifold $\mathrm{X}=[\bullet / G]$.

In 17] we have introduced the string topology of LX. In order to do this we can consider (stack) orbifold correspondences. Such an orbifold correspondence is 
a diagram in the category of orbifolds of the form

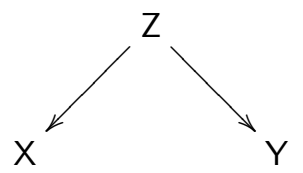

We need of course the concept of smooth degree $-d$ correspondence in the category of infinite dimensional $C^{\infty}$ orbifolds to mimic the arguments of the previous subsections. In any case whenever $\mathbf{X}$ is a $d$ dimensional oriented orbifold, the output of this procedure is a degree $-d$ product on the homology of $\mathrm{LX}$,

$$
H_{*}(\mathrm{LX}) \otimes H_{*}(\mathrm{LX}) \rightarrow H_{*-d}(\mathrm{LX}) .
$$

In 17] we consider mostly global orbifolds, namely orbifolds of the form $\mathrm{X}=$ $[M / G]$ where $G$ acts in an orientation preserving fashion on the compact, closed smooth manifold $M$. In this case we have that $A_{\mathrm{LX}}=H_{*}(\mathrm{LX}) \cong H_{*}\left(\mathcal{L}\left(M \times_{G} E G\right)\right)$. Here the main idea comes from string theory, and it is to consider the elements of $G$ as 0-branes, in the form of boundary conditions for the strings. In any case, using classical algebraic topology we prove the following.

Theorem 9.2.1. Let $\mathrm{X}=[M / G]$, then $A_{\mathrm{LX}} \cong H_{*}\left(\mathcal{L}\left(M \times_{G} E G\right) ; \mathbb{Q}\right)$ has the structure of a Batalin-Vilkovisky algebra, and moreover

- When $G=\{1\}$ and for arbitrary $M$ then $A_{\mathrm{Lx}}$ coincides with the ChasSullivan BV-algebra.

- More interestingly, when $M=\left\{m_{0}\right\}$ is a single point and for arbitrary $G$ then $A_{\mathrm{Lx}}$ coincides with the Dijkgraaf-Witten Frobenius-algebra.

9.3. Chen-Ruan Orbifold Stringy Cohomology. The Chen-Ruan theory 5 is a $(1+1)$-dimensional TQFT whose fields are of the form

$$
\mathcal{F}(Y)=\operatorname{Hol}^{\odot}(Y, \mathrm{X}) .
$$

The constant holomorphic orbifold maps from $Y$ to $\mathrm{X}$. Recall that the localization principle states that for the boundary values we have

$$
\mathcal{F}\left(S^{1}\right)=\operatorname{Map}^{\odot}\left(S^{1}, \mathrm{X}\right)=I(\mathrm{X}),
$$

is the inertia orbifold. The correspondence associated to the pair of pants $P$ is

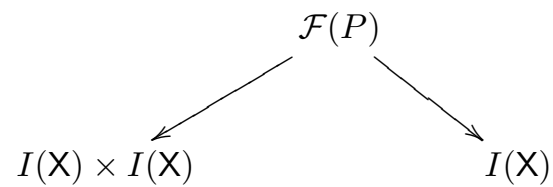

and it gives a product

$$
H^{*}(I(\mathrm{X})) \otimes H^{*}(I(\mathrm{X})) \rightarrow H^{*}(I(\mathrm{X}))
$$

We should mention that $\mathcal{F}(P)$ has a virtual fundamental class of rational degree (called the degree shifting in the terminology of Chen and Ruan). Considering this product, and the degree shift the Chen-Ruan cohomology of an orbifold is defined as

$$
H_{\text {Chen-Ruan }}^{*}(\mathrm{X}):=H^{*}(I(\mathrm{X})) \text {. }
$$

For a survey of its remarkable properties and conjectures we recommend the reader to consult [23, 24. 


\section{Acknowledgments}

This paper is based on the lectures given by the first author in Villa de Leyva, Colombia in August 2005. We would like to thank the organizers of the summer school for the invitation to deliver the lectures.

The authors would like to thank Alejandro Adem, Ralph Cohen, Dan Freed, Graeme Segal and Constantin Teleman for conversations that have clarified diverse aspects of the subjects covered here.

Both authors woud like to thank the MSRI for its hospitality during the preparation of this manuscript, the second author would also like to thank the Max Planck Institut in Bonn.

\section{References}

1. Lowell Abrams, Two-dimensional topological quantum field theories and Frobenius algebras, J. Knot Theory Ramifications 5 (1996), no. 5, 569-587. MR MR1414088 (97j:81292)

2. A. Adem and Y. Ruan, Twisted orbifold K-theory, Comm. Math. Phys. 237 (2003), no. 3, 533-556. MR 1993337

3. Michael Atiyah, Topological quantum field theories, Inst. Hautes Études Sci. Publ. Math. (1988), no. 68, 175-186 (1989). MR MR1001453 (90e:57059)

4. M. Chas and D. Sullivan, String topology, arXiv:math.GT/9911159.

5. Weimin Chen and Yongbin Ruan, A new cohomology theory of orbifold, Comm. Math. Phys. 248 (2004), no. 1, 1-31. MR MR2104605 (2005j:57036)

6. R. Cohen and V. Godin, A polarized view of string topology, arXiv:math.AT/0303003.

7. R. Cohen and J. Jones, A homotopy theoretic realization of string topology, Math. Ann. 324 (2002), no. 4, 773-798. MR 2004c:55019

8. R. Cohen and A. Voronov, Notes on string topology, arXiv:math.GT/0503625.

9. Tommaso de Fernex, Ernesto Lupercio, Thomas Nevins, and Bernardo Uribe, A Localization Principle for Orbifold Theories, arXiv:hep-th/0411037, to appear in Contemporary Mathematics.

10. Robbert Dijkgraaf and Edward Witten, Topological gauge theories and group cohomology, Comm. Math. Phys. 129 (1990), no. 2, 393-429. MR MR1048699 (91g:81133)

11. Richard Feynman, Selected papers of Richard Feynman, World Scientific Series in 20th Century Physics, vol. 27, World Scientific Publishing Co. Inc., River Edge, NJ, 2000, With commentary, Edited by Laurie M. Brown. MR MR1888521 (2002k:01042)

12. E. Getzler, Batalin-Vilkovisky algebras and two-dimensional topological field theories, Comm. Math. Phys. 159 (1994), no. 2, 265-285. MR 95h:81099

13. Maxim Kontsevich, Enumeration of rational curves via torus actions, The moduli space of curves (Texel Island, 1994), Progr. Math., vol. 129, Birkhäuser Boston, Boston, MA, 1995, pp. 335-368. MR MR1363062 (97d:14077)

14. J. L. Koszul, Sur certains groupes de transformations de Lie, Géométrie différentielle. Colloques Internationaux du Centre National de la Recherche Scientifique, Strasbourg, 1953, Centre National de la Recherche Scientifique, Paris, 1953, pp. 137-141. MR MR0059919 (15,600g)

15. Serge Lang, Algebra, third ed., Graduate Texts in Mathematics, vol. 211, Springer-Verlag, New York, 2002. MR MR1878556 (2003e:00003)

16. E. Lupercio and B. Uribe, Loop groupoids, gerbes, and twisted sectors on orbifolds, Orbifolds in mathematics and physics (Madison, WI, 2001), Contemp. Math., vol. 310, Amer. Math. Soc., Providence, RI, 2002, pp. 163-184. MR 1950946

17. E. Lupercio, B. Uribe, and M. Xicoténcatl, Orbifold string topology, arXiv:math.AT/0512658.

18. Ernesto Lupercio and Bernardo Uribe, Inertia orbifolds, configuration spaces and the ghost loop space, Q. J. Math. 55 (2004), no. 2, 185-201. MR MR2068317 (2005f:57032)

19. Saunders Mac Lane, Categories for the working mathematician, second ed., Graduate Texts in Mathematics, vol. 5, Springer-Verlag, New York, 1998. MR MR1712872 (2001j:18001)

20. Ieke Moerdijk, Orbifolds as groupoids: an introduction, Orbifolds in mathematics and physics (Madison, WI, 2001), Contemp. Math., vol. 310, Amer. Math. Soc., Providence, RI, 2002, pp. 205-222. MR MR1950948 (2004c:22003) 
21. S. Piunikhin, D. Salamon, and M. Schwarz, Symplectic Floer-Donaldson theory and quantum cohomology, Contact and symplectic geometry (Cambridge, 1994), Publ. Newton Inst., vol. 8, Cambridge Univ. Press, Cambridge, 1996, pp. 171-200. MR MR1432464 (97m:57053)

22. Yongbin Ruan, Topological sigma model and Donaldson-type invariants in Gromov theory, Duke Math. J. 83 (1996), no. 2, 461-500. MR MR1390655 (97d:58042)

23. Stringy geometry and topology of orbifolds, Symposium in Honor of C. H. Clemens (Salt Lake City, UT, 2000), Contemp. Math., vol. 312, Amer. Math. Soc., Providence, RI, 2002, pp. 187-233. MR MR1941583 (2004b:32051)

24. - Stringy orbifolds, Orbifolds in mathematics and physics (Madison, WI, 2001), Contemp. Math., vol. 310, Amer. Math. Soc., Providence, RI, 2002, pp. 259-299. MR MR1950951 (2004e:53136)

25. I. Satake, The Gauss-Bonnet theorem for $V$-manifolds, J. Math. Soc. Japan 9 (1957), 464-492. MR 20 \#2022

26. G. Segal, Classifying spaces and spectral sequences, Inst. Hautes Etudes Sci. Publ. Math. 34 (1968), 105-112.

27. Graeme Segal, The Stanford Lectures on QFT.

Departamento de Matemáticas, CinvestaV, Apartado Postal 14-740 México, D.F. 07000 MÉXICO

E-mail address: lupercio@math.cinvestav.mx

Departamento de Matemáticas, Universidad de los Andes, Carrera 1 N. 18A - 10, Bogotá, COLOMBIA

E-mail address: buribe@uniandes.edu.co 